\title{
Editorial
}

\section{Nanomaterials for Sensor Device Applications}

\author{
Liang-Wen Ji, ${ }^{1}$ Sheng-Joue Young, ${ }^{2}$ Chih-Hung Hsiao, ${ }^{3}$ and Artde Donald Kin-Tak Lam ${ }^{4}$ \\ ${ }^{1}$ Institute of Electro-Optical and Materials Science, National Formosa University, Yunlin County 632, Taiwan \\ ${ }^{2}$ Department of Electronic Engineering, National Formosa University, Yunlin County 632, Taiwan \\ ${ }^{3}$ Institute of Microelectronics and Department of Electrical Engineering, Center for Micro/Nano Science and Technology, \\ Advanced Optoelectronic Technology Center, National Cheng Kung University, Tainan 701, Taiwan \\ ${ }^{4}$ Institute of Creative Industry Research, Fuzhou University, Fuzhou, Fujian 350108, China \\ Correspondence should be addressed to Liang-Wen Ji; lwji@seed.net.tw
}

Received 27 March 2014; Accepted 27 March 2014; Published 10 April 2014

Copyright (C) 2014 Liang-Wen Ji et al. This is an open access article distributed under the Creative Commons Attribution License, which permits unrestricted use, distribution, and reproduction in any medium, provided the original work is properly cited.

Nanostructures can be divided into zero-dimensional (0D when they are quantum dot or nanoparticle structures), one-dimensional (1D when they are elongated), and twodimensional (2D when they are planar) based on their shapes. Nanostructured materials are a new class of materials, having dimensions in the $1 \sim 100 \mathrm{~nm}$ range, which provide one of the greatest potentials for improving performance and extended capabilities of products in a number of industrial sectors. In recent years, the nanostructures for sensor device applications have been highly developed in various fields, due to their flexibility and light weight for daily use. Some materials like the low carbon alloy steel, transparency conducting AZO films, piezoelectric material, AlN flake, and titanium dioxide films have already been introduced and under investigation. These materials and devices often have unknown reliability behavior and/or introduce new failure mechanisms. In addition, the market is continuously demanding higher reliability levels for present technologies. The chance to share and discuss these crucial nanostructured materials for sensor device developments is very important. Therefore, the field of sensor device has been the subject of reviews. Sensor devices are environmentally sustainable, in particularly considering the availability of the nanostructured raw materials.

This special issue selects 22 papers about nanomaterials for sensor device applications and other related fields. This special issue enables interdisciplinary collaboration between material science and engineering technologists in the academic and industrial fields.

Liang-Wen Ji

Sheng-Joue Young

Chih-Hung Hsiao

Artde Donald Kin-Tak Lam 

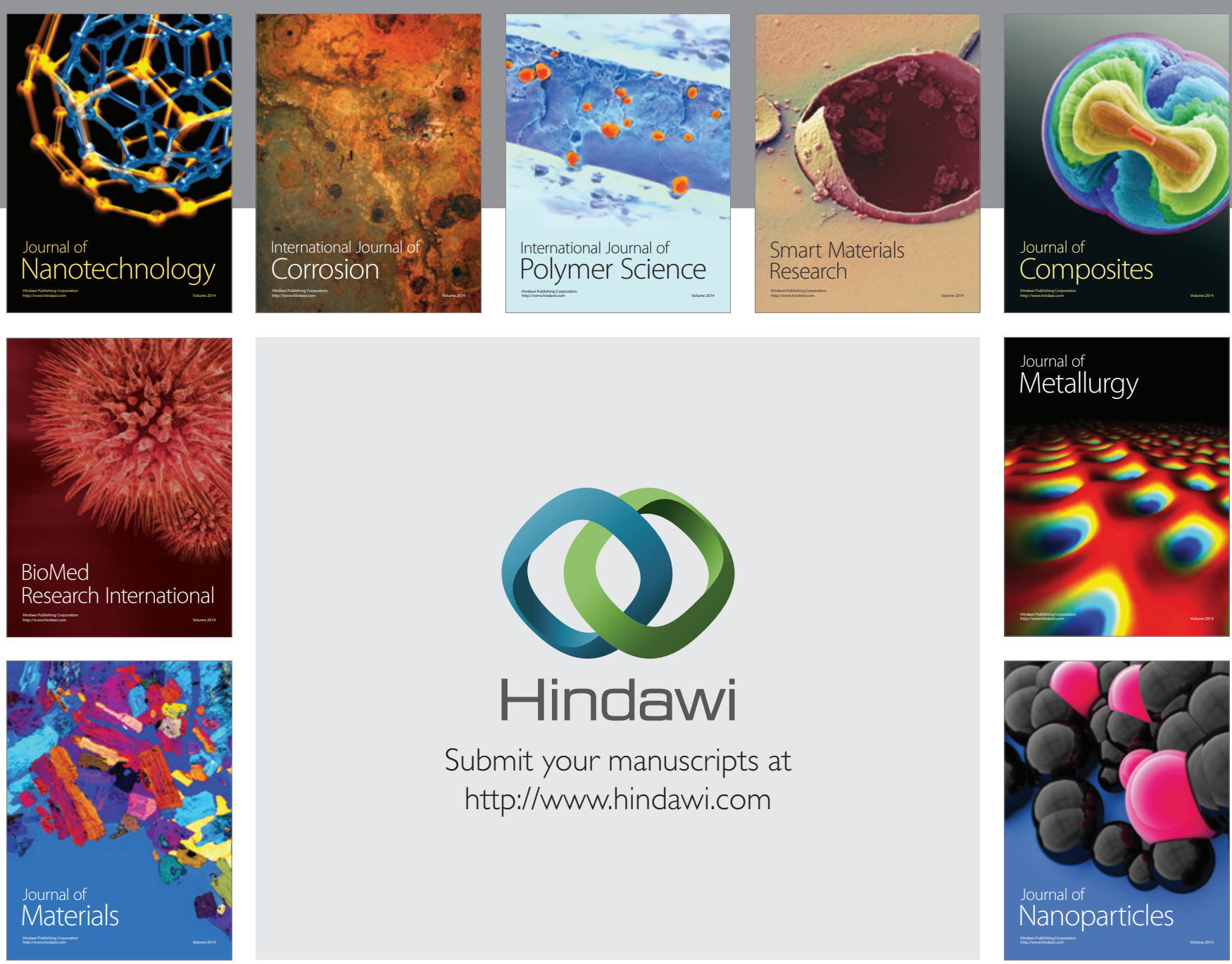

Submit your manuscripts at http://www.hindawi.com
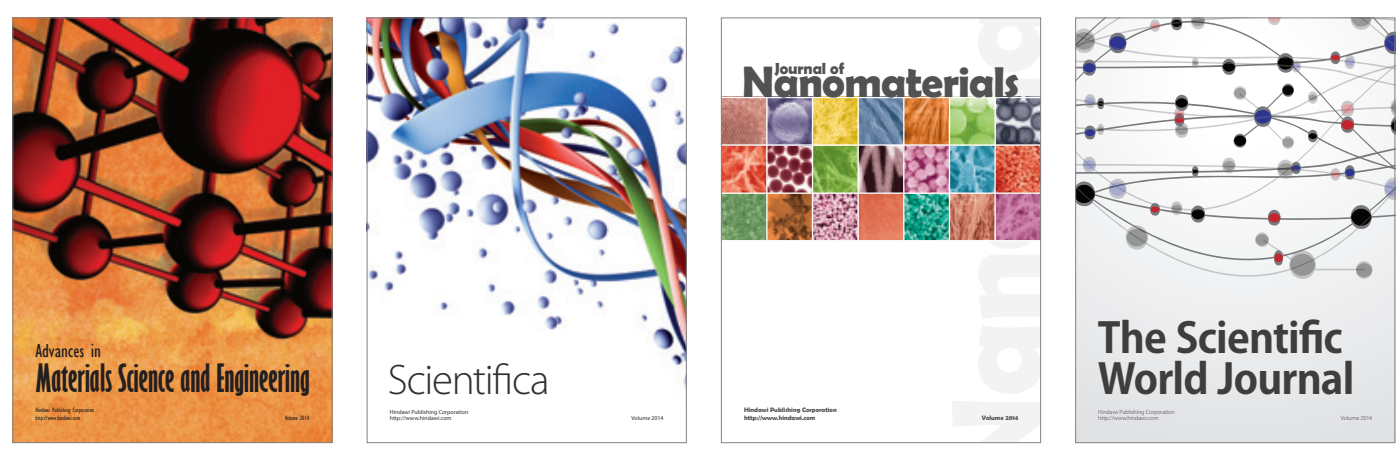

\section{The Scientific World Journal}
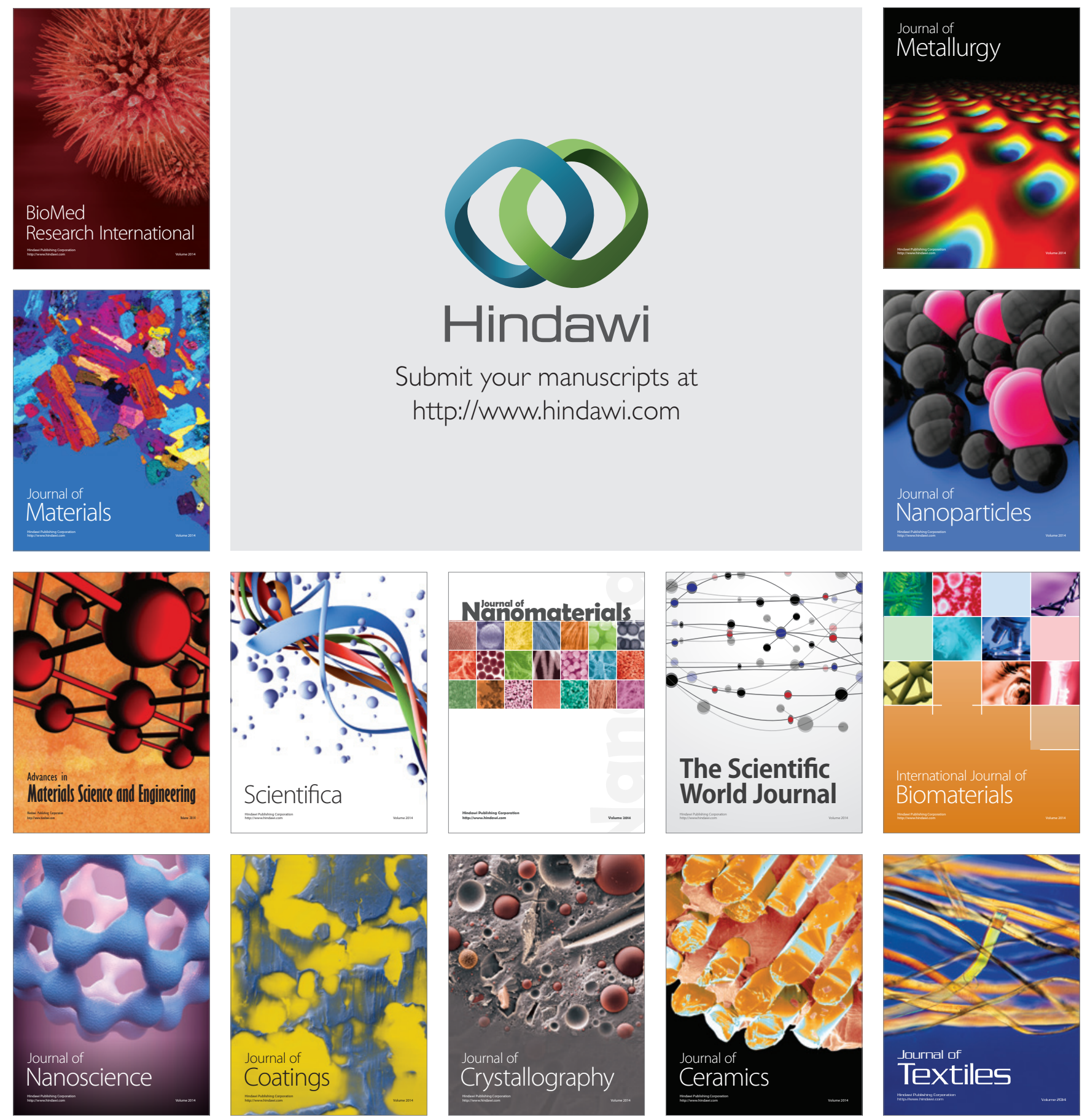